\title{
In vivo short-term and long-term host reaction to starch-based scaffolds
}

\author{
T.C. Santos ${ }^{\mathrm{a}, \mathrm{b}, *}$, A.P. Marques ${ }^{\mathrm{a}, \mathrm{b}}$, B. Höring ${ }^{\mathrm{c}}$, A.R. Martins $^{\mathrm{a}, \mathrm{b}}$, K. Tuzlakoglu $^{\mathrm{a}, \mathrm{b}}$, A.G. Castro ${ }^{\mathrm{d}}$, \\ M. van Griensven ${ }^{\text {c }}$, R.L. Reis ${ }^{\mathrm{a}, \mathrm{b}}$ \\ a 3B's Research Group-Biomaterials, Biodegradables and Biomimetics, University of Minho, Headquarters of the European Institute of Excellence on Tissue Engineering \\ and Regenerative Medicine, AvePark, 4806-909 Taipas, Guimarães, Portugal \\ ${ }^{\mathrm{b}}$ IBB-Institute for Biotechnology and Bioengineering, PT Associated Laboratory, Guimarães, Portugal \\ ${ }^{\mathrm{c}}$ Ludwig Boltzmann Institute for Experimental and Clinical Traumatology, Austrian Cluster for Tissue Regeneration, Donaueschingenstrasse 13, A-1200 Vienna, Austria \\ ${ }^{\mathrm{d}}$ Life and Health Sciences Research Institute (ICVS), School of Health Sciences, University of Minho, 4710-057 Braga, Portugal
}

\section{A R T I C L E I N F O}

\section{Article history:}

Received 25 February 2010

Received in revised form 25 May 2010

Accepted 15 June 2010

Available online 23 June 2010

\section{Keywords:}

Starch-based scaffolds

Host reaction

Subcutaneous implantation

Intramuscular implantation

Natural polymers

\begin{abstract}
A B S T R A C T
The implantation of biomaterials may elicit a host response to this foreign body, and the magnitude of that reaction depends on the host and on the implanted material. The aim of this study was to compare the inflammatory response induced by the implantation of starch-based (SPCL) scaffolds in two implantation rat models: subcutaneous (SC) and intramuscular (IM). Moreover, two methodologies, wet spinning (WS) and fibre-bonding (FB), were used to prepare the scaffolds. The short-term inflammatory/ immune host reaction was assessed by SC and IM implantations in rats after 1 and 2 weeks, and the long-term host response was addressed after 8 and 12 weeks of SC implantation of both types of SPCL scaffolds in rats. After each time period, the scaffolds, surrounding tissue and nearby lymph nodes were explanted, and used for histological analysis and molecular biology evaluation. The results showed that SPCL-WS scaffolds seem to induce a slight lower inflammatory/immune reaction in both types of implantation models. Nonetheless, comparing the two models, the IM implantation resulted in a slightly higher inflammatory response than the SC implantation with early activation of the lymph nodes. The overall data suggests a good integration of the materials in the host, independently of the tissue location with a normal progress of the reaction for all the conditions.
\end{abstract}

(c) 2010 Acta Materialia Inc. Published by Elsevier Ltd. All rights reserved.

\section{Introduction}

The constant development of biomaterials in the field of tissue engineering (TE) is an attempt to meet the rising demands of new tissue replacement/regeneration strategies. However, the increasing complexity of the TE devices, comprising cells [1-6] and/or bioactive agents [7-10] within 3-D scaffolding structures, involves additional concerns regarding adverse host reactions to the implantable constructs [11]. A considerable number of studies [12-17] have demonstrated that the immunomodulatory properties of mesenchymal stem cells obtained from different sources seem to circumvent a potential host rejection of the transplanted cells.

The incorporation of foreign growth factors, eventually considered as immunogenic, in TE constructs is nowadays a recurrent approach as researchers have concentrated on recombinant tech-

\footnotetext{
* Corresponding author at: 3B's Research Group-Biomaterials, Biodegradables and Biomimetics, University of Minho, Headquarters of the European Institute of Excellence on Tissue Engineering and Regenerative Medicine, AvePark, 4806-909 Taipas, Guimarães, Portugal. Tel.: +351 253 510913; fax: +351 253510909.

E-mail address: tircia.santos@dep.uminho.pt (T.C. Santos).
}

nology to produce recombinant bioactive molecules $[8,9,18]$, which play a key role in tissue regeneration.

It seems that, despite all the investment in the research on stem cell technology, as well as in the identification of key mediators in the inflammation/immune reaction and differentiation pathways in the field of TE, the role of scaffolds, as simple supports, in the specific host reaction has been neglected. Natural-origin biomaterials have been considered for many years as a way to improve, in comparison to synthetic polymers, in vivo biofunctionality and to modulate/avoid a harmful host response due to their similarities with biological molecules. Starch-based scaffolds, processed using several methodologies aiming at a range of TE applications [19-26], have demonstrated great potential in this field. Very promising results for bone tissue regeneration have in particular been obtained with a blend of starch and polycaprolactone (SPCL) [20-23,25,27-30]. SPCL scaffolds, with adequate physicochemical and mechanical properties for bone TE $[19,22]$ and adequate degradability rate [24,25,31], have been shown to support mesenchymal stem cell growth and differentiation [19,20], and to be excellent supporting structures for endothelial cells [22,23,32,33]. However, a lacuna is still present concerning the in vivo reaction of SPCLbased scaffolds. A systematic study was carried out using two 
different rat implantation models, subcutaneous (SC) and intramuscular (IM). The aim of the study was (i) to understand the tissue reaction to two SPCL-based scaffolds produced by different methodologies, wet spinning [26] and fibre-bonding [22], for both short- and long-term implantation periods; and (ii) to identify eventual differences between the two models in terms of inflammatory/immune response elicited by the two different forms of SPCL-based scaffolds.

\section{Materials and methods}

\subsection{Materials}

Starch-based scaffolds of SPCL were produced from a blend of starch with $\beta$-polycaprolactone (30:70\%), by two different methodologies described elsewhere: wet spinning (SPCL-WS) [26] and fibre-bonding (SPCL-FB) [22]. Briefly, for the production of SPCL-WS scaffolds, the polymer was dissolved in chloroform at a concentration of $40 \%(\mathrm{w} / \mathrm{v})$ in order to obtain a polymer solution with proper viscosity. The polymer solution was loaded into a syringe, which was placed in a syringe pump (World Precision Instruments, UK) and a certain amount of polymer solution was subsequently extruded into a methanol coagulation solution. The fibre mesh structure was formed during the processing by the random movement of the precipitation container. The formed scaffolds were then dried overnight at room temperature to allow any remaining solvents to evaporate. For the fabrication of the SPCL-FB scaffolds, fibre-meshes previously obtained by a meltspinning methodology were placed in a glass mould and heated in an oven at $150^{\circ} \mathrm{C}$. Immediately after removing the moulds from the oven, the fibres were slightly compressed by a Teflon cylinder and then cooled at $-15^{\circ} \mathrm{C}$. All samples were cut into discs of $5 \mathrm{~mm}$ diameter and approximately $1 \mathrm{~mm}$ thickness and sterilized by a standard procedure with ethylene oxide [34].

\subsection{Intramuscular implantation}

Six male Sprague-Dawley rats weighing between 380 and $400 \mathrm{~g}$ (three for each implantation time period of 1 and 2 weeks) were used. Each animal was anaesthetized with an IM injection of $90 \mathrm{mg} \mathrm{kg}^{-1}$ ketamine hydrochloride and $5 \mathrm{mg} \mathrm{kg}^{-1}$ xylazine hydrochloride. After shaving and disinfecting the back of the animals, four paravertebral skin incisions, of approximately $2 \mathrm{~cm}$, containing the subcutis and the panniculus carnosus, at the level of the left and right scalenus dorsalis and gluteus muscles, respectively, were performed under sterile surgical conditions. An incision of the fascia of the muscles was performed and craniolateral oriented muscle-pockets were created by blunt dissection. After introducing the scaffolds (four scaffolds per animal), previously kept in a sterile saline solution, the fascia, the panniculus carnosus and finally the skin were carefully sutured. The animals were kept in single cages with food and water ad libitum throughout the time of implantation. During the first week, the animals received daily $200 \mu \mathrm{g} \mathrm{g}^{-1}$ body weight of metamizole sodium in their drinking water.

\subsection{Subcutaneous implantation}

Six male Sprague-Dawley rats weighing between 350 and $380 \mathrm{~g}$ (three for each implantation time period of 1 and 2 weeks), were used. Each test animal was anaesthetized with an IM injection of $90 \mathrm{mg} \mathrm{kg}^{-1}$ ketamine hydrochloride and $5 \mathrm{mg} \mathrm{kg}^{-1}$ xylazine hydrochloride and two medial and rostral incisions of approximately $2 \mathrm{~cm}$ containing the subcutis and the panniculus carnosus were performed in the dorsum of the rats. Craniolateral oriented pockets (two per incision) were subcutaneously created by blunt dissection. The scaffolds (four scaffolds per animal), previously kept in a sterile saline solution, were introduced into the pockets and the panniculus carnosus and the skin were carefully sutured. The animals were kept in single cages with food and water ad libitum during all time of implantation. During the first week, the animals received daily $200 \mu \mathrm{g} \mathrm{g}^{-1}$ of body weight of metamizole sodium in their drinking water.

For the long-term reaction, six male Sprague-Dawley rats (three for each implantation time period of 8 and 12 weeks), weighing between 280 and $340 \mathrm{~g}$ were used for the SC implantation of the SPCL scaffolds. The surgical procedure followed was the same as described mentioned for the IM implantation.

\subsection{Post-implantation analysis}

At the end point times (1, 2, 8 and 12 weeks), each animal was IM anaesthetized as described above and killed with an intracardial overdose of $90 \mathrm{mg} \mathrm{kg}^{-1}$ ketamine hydrochloride and $5 \mathrm{mg} \mathrm{kg}^{-1}$ xylazine hydrochloride. From each animal the four implanted scaffolds and surrounding tissue, as well as the axillary and inguinal lymph nodes, were explanted. The explanted samples were either fixed in 3.7\% formalin for histological evaluation, or frozen for posterior molecular biology analysis. The histological analysis of crosssection samples was performed after haematoxylin and eosin (HE), and Masson Goldner Trichrome (MGT) staining and immunohistochemistry using a monoclonal mouse anti-human CD3 antibody (Dako, Denmark) with cross-reactivity for rat $\mathrm{T}$ lymphocytes, a monoclonal mouse anti-rat CD18 antibody (Serotec, UK) for the integrin $\beta 2$ chain of recruited leukocytes, and a monoclonal mouse anti-human phosphoinositide 3-Kinase (Pi3K) antibody (BD, Belgium) with cross-reactivity for rat activated and proliferating lymphocytes, following standard protocols. Reverse transcriptase polymerase chain reaction (RT-PCR) to detect the expression of

Table 1

Forward and reverse sequences of the genes detected by RT-PCR on rat samples.

\begin{tabular}{|c|c|c|c|c|}
\hline Function & Gene & Sequences & $\begin{array}{l}T_{\mathrm{m}} \\
\left({ }^{\circ} \mathrm{C}\right)\end{array}$ & Bp \\
\hline Housekeeping gene & GAPDH & $\begin{array}{l}\text { Sense- } \\
\text { GGTGATGCTGGTGCTGAGTA } \\
\text { Antisense- } \\
\text { GGATGCAGGGATGATGTTCT }\end{array}$ & $\begin{array}{l}59.4 \\
57.3\end{array}$ & 81 \\
\hline \multirow[t]{2}{*}{ Pro-inflammatory } & IL-18 & $\begin{array}{l}\text { Sense- } \\
\text { AGATGTGGAACTGGCAGAGG } \\
\text { Antisense- } \\
\text { CCCATTTGGGAACTTCTCCT }\end{array}$ & $\begin{array}{l}59.4 \\
57.3\end{array}$ & 220 \\
\hline & IL-1 $\alpha$ & $\begin{array}{l}\text { Sense- } \\
\text { GCAAAGCCTAGTGGAACCAG } \\
\text { Antisense- } \\
\text { GCAGAAGGTGCACAGTGAGA }\end{array}$ & $\begin{array}{l}59.4 \\
59.4\end{array}$ & 244 \\
\hline \multirow[t]{4}{*}{ Anti-inflammatory } & IL-10 & $\begin{array}{l}\text { Sense- } \\
\text { GAATTCCCTGGGAGAGAAGC } \\
\text { Antisense- } \\
\text { CCGGGTGGTTCAATTTTTCAT }\end{array}$ & $\begin{array}{l}59.4 \\
55.9\end{array}$ & 219 \\
\hline & IL-13 & $\begin{array}{l}\text { Sense- } \\
\text { ATCGAGGAGCTGAGCAACAT } \\
\text { Antisense- } \\
\text { CGAGGCCTTTTGGTTACAGA }\end{array}$ & $\begin{array}{l}57.3 \\
57.3\end{array}$ & 189 \\
\hline & IFN- $\gamma$ & $\begin{array}{l}\text { Sense- } \\
\text { GCCCTCTCTGGCTGTTACTG } \\
\text { Antisense- } \\
\text { CTGATGGCCTGGTTGTCTTT }\end{array}$ & $\begin{array}{l}61.4 \\
57.3\end{array}$ & 221 \\
\hline & $\begin{array}{l}\text { MHC } \\
\text { class II }\end{array}$ & $\begin{array}{l}\text { Sense- } \\
\text { TCCCAGATACACAGCAGCAG } \\
\text { Antisense- } \\
\text { CATGCGAAGGTTCTCCAGTT }\end{array}$ & $\begin{array}{l}59.4 \\
57.3\end{array}$ & 320 \\
\hline
\end{tabular}


IL-1 $\alpha$, IL-18, IL-10, IL-13, IFN- $\gamma$ and MHC class II genes was carried out. The detailed description of the genes is summarized in Table 1. Image analysis of the histological sections of the 8 and 12 week explants, considering the scaffold and the inflammation areas, was performed with Olympus CellP software (Olympus, Belgium) and using an Olympus BX61 microscope (Olympus, Belgium). The methodology to create the areas of interest was followed according to the software instructions.

\subsection{Statistical analysis}

Mean values and standard deviations are reported for the measurements [35] of the scaffold and the surrounding inflammation areas. Data was analysed by a single-factor ANOVA test and the significance value was set at $P<0.05$.

\section{Results}

\subsection{Intramuscular implantation}

Macroscopic signs of inflammation, infection or swelling were absent after 1 and 2 weeks of IM implantation of the different types of SPCL scaffolds.

The histological analysis of the explanted materials and respective surrounding tissue revealed the absence of oedema and necrosis both at 1 and 2 weeks of implantation (Fig. 1A, C, E and G). After the first week of IM implantation of SPCL-FB scaffolds, the inflammatory infiltrate around the scaffold fibres (Fig. 1A) mainly comprised polymorphonuclear neutrophils (PMNs), characterized by their multilobular nuclei. The presence of recruited leukocytes, CD18-positive cells, was confirmed by immunohistochemistry using the specific marker of $\beta 2$ integrin (Fig. 2A). The analysis carried out for the SPCL-WS scaffolds implanted IM seemed to indicate a reduction in the amount of inflammatory infiltrate (Fig. 1C) in comparison to what was observed for the SPCL-FB (Fig. 1A), although PMNs were also present at the surrounding area of the scaffold's fibres (Fig. 2C). At this early stage of implantation it was noticed that, for both SPCL-WS and SPCL-FB scaffolds, some collagen network started to be deposited between the scaffold's fibres, as shown by MGT staining (Fig. 1B and D).

After the second week of implantation, the nature of the observed inflammatory infiltrate in the tissue surrounding the SPCL-FB scaffolds implanted IM was different than the observed at the first week of implantation. Mononuclear cells (Fig. 1E), namely $\mathrm{T}$ lymphocytes, as indicated by their positivity in the CD3 marker (Fig. 2E), were predominant. Similarly, T lymphocytes were also present in the tissue surrounding the SPCL-WS fibres (Fig. 2G). Additionally, some foreign body giant cells (FBGCs) appeared at the fibre interfaces of both the SPCL-WS and the SPCL-FB scaffolds (Fig. 1E and G). In comparison to the first week of implantation, a denser network of collagen fibres was also observed 2 weeks after the IM implantation of both types of SPCL scaffolds (Fig. 1F and $\mathrm{H}$ ). After 2 weeks of implantation, a significant amount of blood vessels was also observed within the tissue surrounding the fibres of both SPCL scaffolds (Fig. 1E-H).

In order to evaluate an eventual systemic host response to the intramuscular implantation of the different types of SPCL scaffolds, the axillary and inguinal lymph nodes were analysed. The general structure of the lymph nodes, assessed after HE staining, revealed the presence of germinal centres (lighter area), populated mainly with activated B lymphocytes, and some plasma cells (Fig. 3A and $C$ ) in the cortex of the nodes after 1 week of implantation of both SPCL scaffolds. Additionally, denser areas surrounding the germinal centres comprehending lymphocytes, which are characterized by the intense blue nuclei staining (Fig. $3 \mathrm{~A}$ and $\mathrm{C}$ ), were ob- served. A specific assessment of cell proliferation was carried out by tracking the signal transduction molecule PI3K. Very few cells were positive for PI3K after 1 week of IM implantation of both types of SPCL scaffolds. After the second week of implantation, the explanted lymph nodes still revealed the presence of germinal centres, with no differences between the two different types of scaffolds (Fig. 3E and G). The immunolabelling of PI3K showed a low number of positive cells for the SPCL-FB scaffolds and no labelling for the SPCL-WS scaffolds (Fig. $3 \mathrm{~F}$ and $\mathrm{H}$ ).

The molecular biology analysis for specific genes of inflammation showed that, both after 1 and 2 weeks, the IM implantation of SPCL-WS and SPCL-FB induced the expression of the inflammatory cytokines IL-18 and IL- $1 \alpha$ (Table 2 ), as well as the anti-inflammatory cytokines IL-10 (Fig. 8 and Table 2) and IFN- $\gamma$ (Table 2). In the same manner, MHC class II was also expressed at both time periods of implantation and for the different types of SPCL scaffolds (Table 2). The other anti-inflammatory cytokine, IL-13, was expressed in all conditions tested except after 2 weeks of implantation of the SPCL-FB scaffolds (Table 2).

\subsection{Subcutaneous implantation}

Macroscopic signs of inflammation, infection or swelling were absent at the end points of the subcutaneous implantation of the SPCL-WS and SPCL-FB scaffolds. The nonexistence of oedema and necrosis was also histologically confirmed (Fig. 4A, C, E and G) for all conditions. A moderate inflammatory infiltrate, essentially characterized by the presence of PMNs (Fig. 4A and C), was observed 1 week after subcutaneous implantation of both SPCL-WS and SPCL-FB scaffolds. However, the intensity of the observed inflammation appeared to be diminished in comparison to what was observed in the first week of IM implantation, in particular for the SPCL-FB scaffold (Fig. 4A and C and Fig. 1A and C). CD18 immunodetection confirmed the presence of mainly recruited PMNs, also identified by the multilobulated shape of the nuclei, after the first week of subcutaneous implantation of both types of SPCL scaffolds (Fig. 5A and C). Similarly to what was observed for the IM implantation of SPCL scaffolds, after 1 week of reaction, a collagen network had begun to be deposited between the scaffold's fibres, although at an apparent lower density (Fig. 4B and D).

Two weeks after SC implantation of the SPCL scaffolds, the nature of the inflammatory infiltrate changed in comparison to the first week of implantation. Some mononuclear cells and FBGCs were observed in the vicinity of the scaffold's fibres (Fig. 4E and G). This tendency was observed for both SPCL-WS and SPCL-FB scaffolds, although the FBGC density seemed to be greater at the SPCL-FB scaffold's interface (Fig. 4G). Mononuclear cells were confirmed to be essentially $\mathrm{T}$ lymphocytes, positive for the CD3 marker, both in SPCL-WS and SPCL-FB (Fig. 5E and G). After 2 weeks, a considerable amount of blood vessels was observed within the tissue surrounding the fibres of the SC implanted scaffolds. As for the IM implantation, the collagen network became more organized (Fig. 4F and $\mathrm{H}$ ) on the outline of the fibres 2 weeks after the SC implantation of both types of SPCL scaffolds.

In terms of systemic reaction, the overall observation of the lymph node structures revealed that, 1 week after SC implantation of both SPCL-WS and SPCL-FB, germinal centres (lighter area) were present in the cortex of the nodes (Fig. $6 \mathrm{~A}$ and $\mathrm{C}$ ). Compared to the IM implantation of SPCL scaffolds, denser areas surrounding the germinal centres of the lymph nodes containing lymphocytes (Fig. 6A and C) were also observed. PI3K was not detected in the lymph nodes of the animals with SPCL-WS SC implanted for 1 week (Fig. 6D). Although positively labelled for the SPCL-FB, only few PI3K-positive cells were observed in the first week of SC implantation (Fig. 6B). Two weeks after implantation, the explanted lymph nodes still revealed the presence of germinal centres, with no 

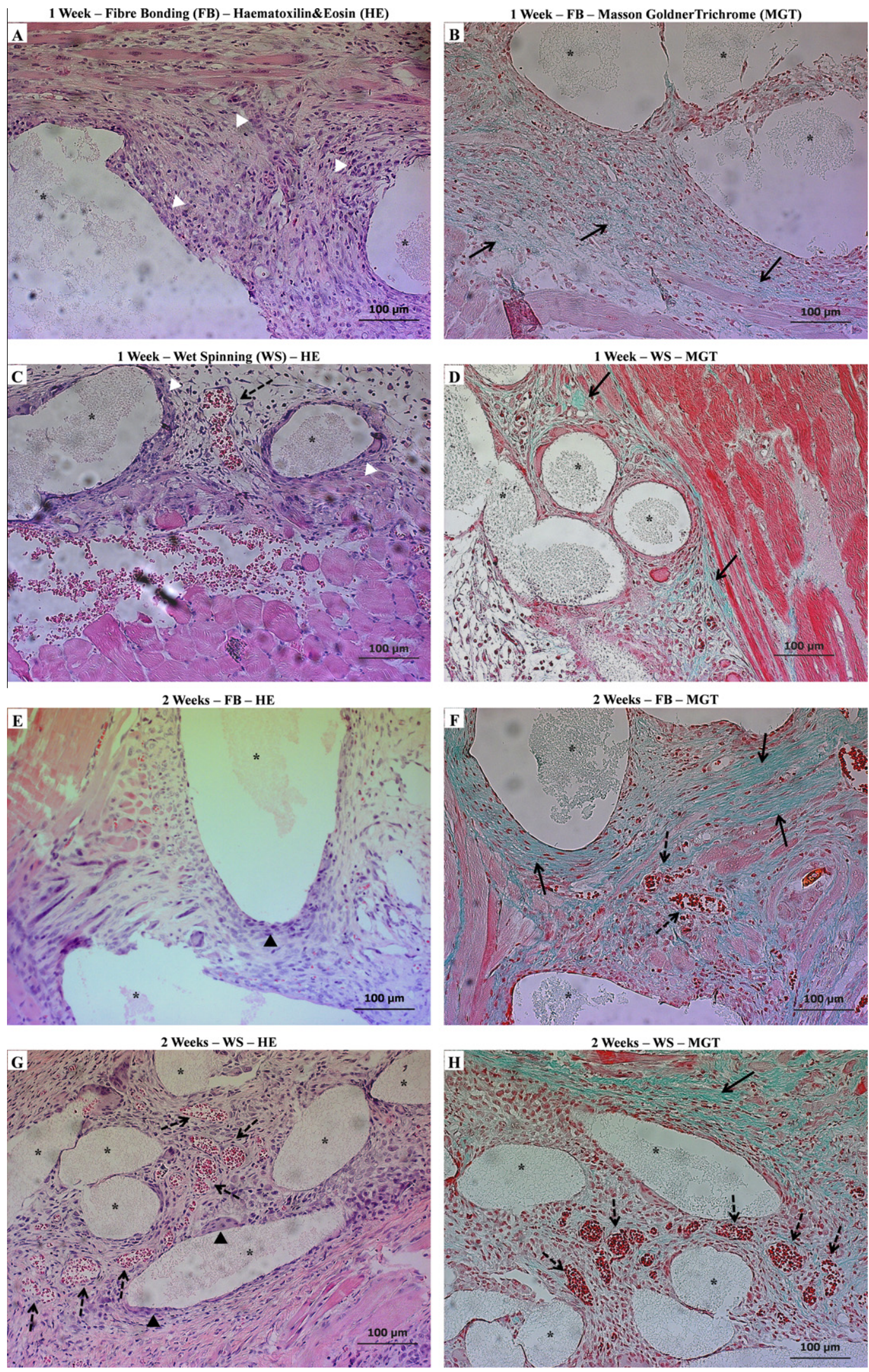

Fig. 1. Micrographs of the sections of the explanted fibre-bonding and wet-spinning produced starch-based scaffolds, after 1 (A-D) and 2 (E-H) weeks of IM implantation in rats. Tissue was stained with HE (A, C, E and G) and MGT (B, D, F and G). * represents the SPCL scaffold fibres. Inflammatory cells are marked with arrowheads, collagen fibres with arrows and blood vessels with dashed arrows.

differences between the animals implanted with the two types of scaffolds (Fig. 6E and G). Again, the PI3K signal was only present in a small number of cells in the nodes of the animals with both types of SPCL scaffolds implanted for 2 weeks (Fig. $6 \mathrm{~F}$ and H). 

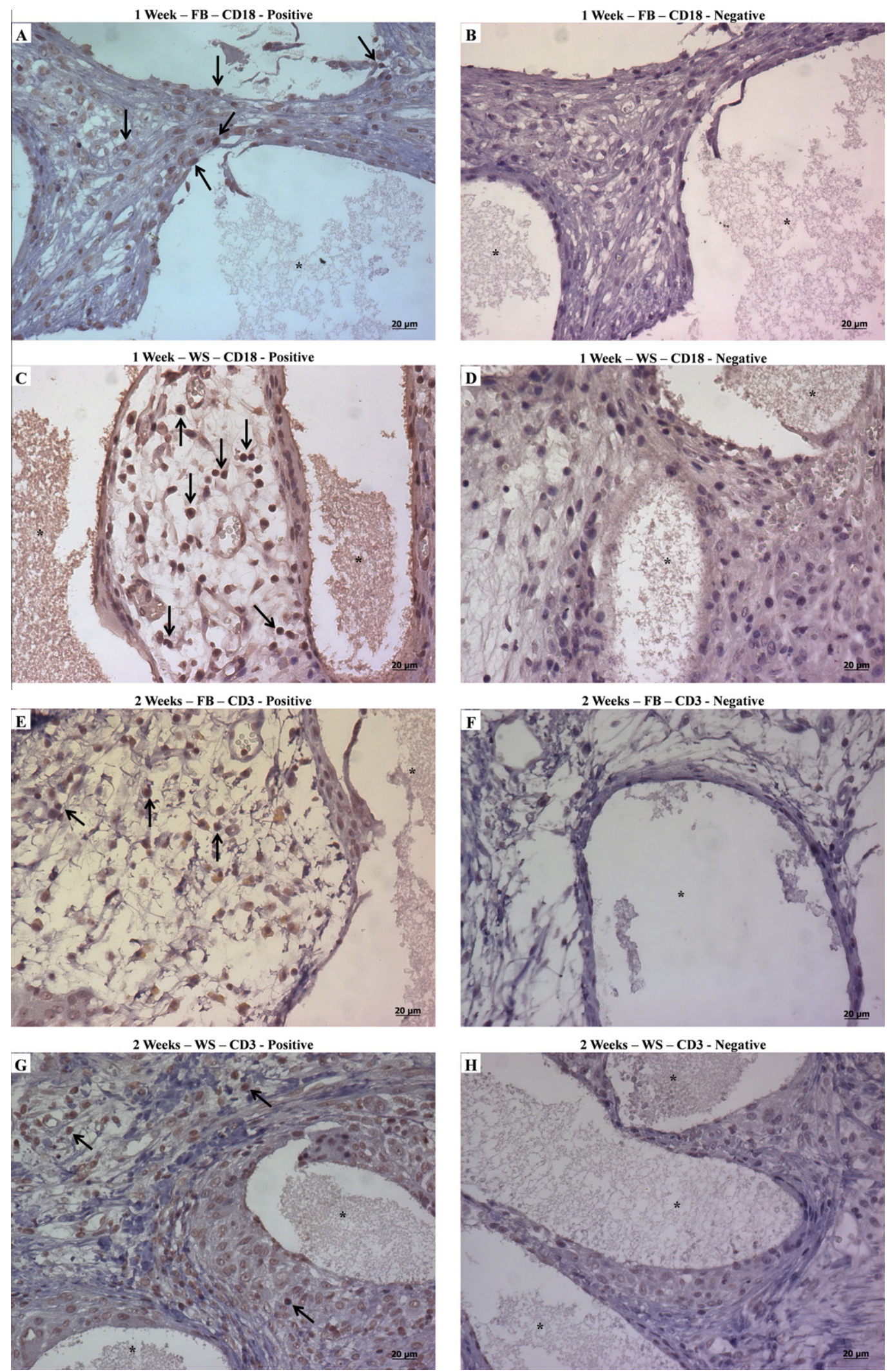

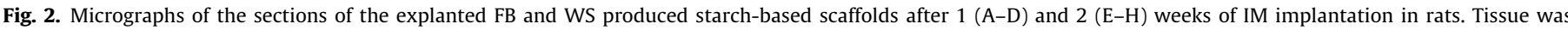
immunohistochemically labelled for CD18 and CD3. * represents the SPCL scaffold fibres, arrows mark CD18-positive cells and dashed arrows mark CD3-positive cells.

The evaluation of a long-term host response to the implantation of SPCL-FB and SPCL-WS scaffolds was performed after 8 and 12 weeks of SC implantation.
The macroscopic features observed at these time points were similar to the ones found for the short-term implantation periods. In fact, the implantation sites did not show visible signs of inflam- 

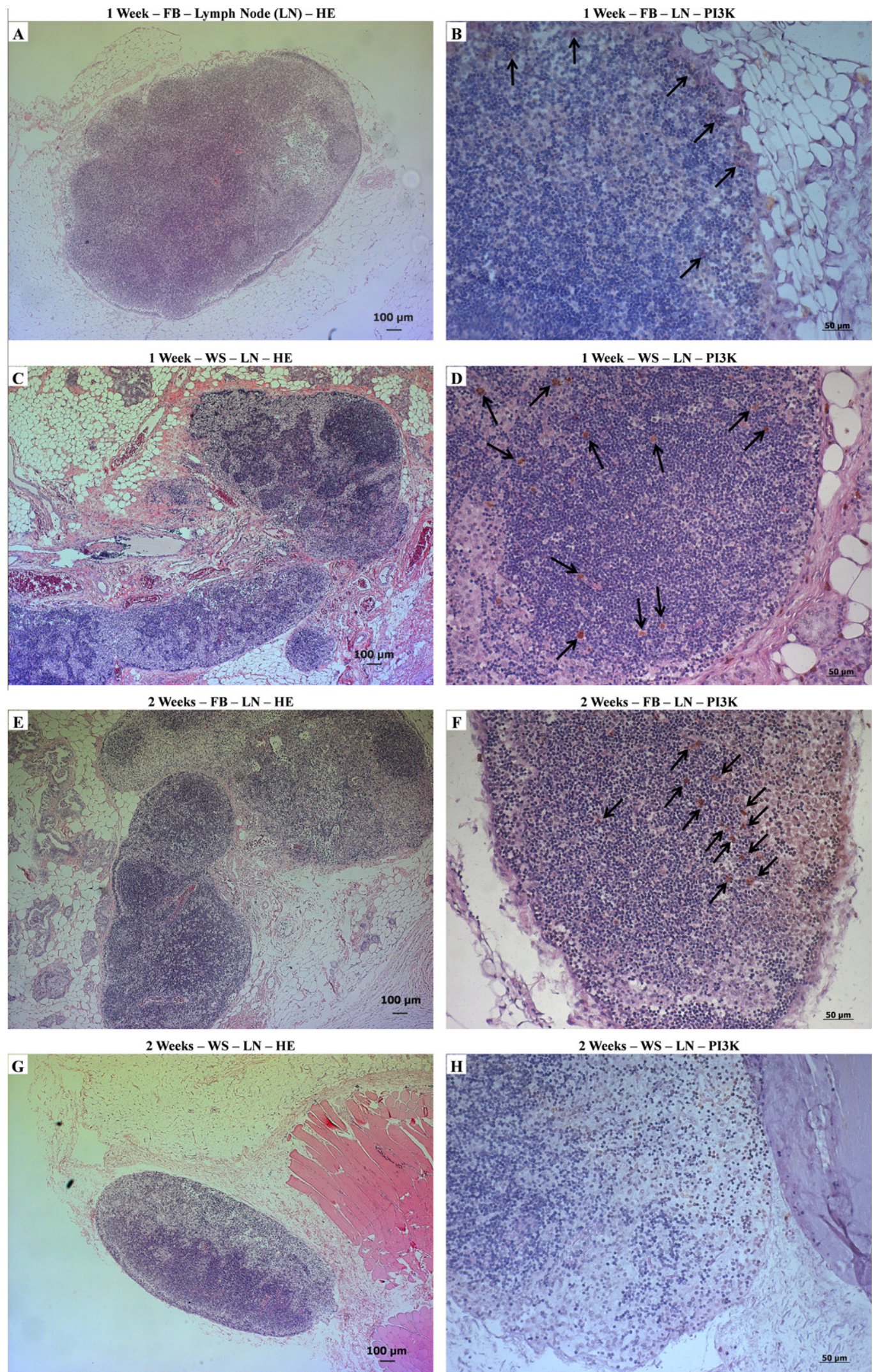

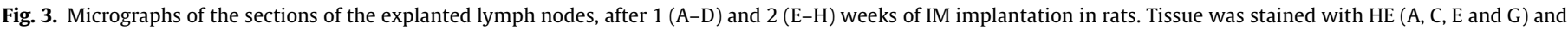
immunohistochemically labelled for Pi3K (B, D, F and G). Arrows indicate positive labelled cells for the PI3K transduction factor.

mation, infection or swelling. At 8 weeks of implantation the histological observation revealed a similar reaction for both SPCL scaf- folds (Fig. 7A and C). At this time point, the inflammatory infiltrate was reduced in comparison to the short-term SC implan- 
Table 2

Presence (+) or absence (-) of expression of the genes detected by RT-PCR on rat samples.

\begin{tabular}{|c|c|c|c|c|c|c|c|}
\hline & GAPDH & IL-18 & IL- $1 \alpha$ & IL-10 & IL-13 & IFN- $\gamma$ & MHC II \\
\hline 7D-SC-WS & + & - & + & - & - & - & - \\
\hline 7D-SC-FB & + & - & + & - & - & - & - \\
\hline 7D-IM-WS & + & + & + & + & - & + & + \\
\hline 7D-IM-FB & + & + & + & + & - & + & + \\
\hline 14D-SC-WS & + & - & + & - & - & - & $\begin{array}{l}- \\
+\end{array}$ \\
\hline 14D-SC-FB & + & - & + & - & - & - & - \\
\hline 14D-IM-WS & + & + & + & + & - & + & + \\
\hline $14 \mathrm{D}-\mathrm{IM}-\mathrm{FB}$ & + & + & + & + & + & + & + \\
\hline 8W-SC-WS & + & - & + & + & + & + & + \\
\hline $8 \mathrm{~W}-\mathrm{SC}-\mathrm{FB}$ & $\begin{array}{l}+ \\
+\end{array}$ & + & + & + & $\begin{array}{l}+ \\
-\end{array}$ & $\begin{array}{l}+ \\
-\end{array}$ & + \\
\hline $12 \mathrm{~W}-\mathrm{SC}-\mathrm{WS}$ & + & - & + & + & + & $\begin{array}{l}+ \\
-\end{array}$ & + \\
\hline $12 \mathrm{~W}-\mathrm{SC}-\mathrm{FB}$ & + & - & + & - & + & + & + \\
\hline
\end{tabular}

tation time periods ( 1 and 2 weeks). No significant differences were observed between 8 and 12 weeks of implantation of the two types of SPCL scaffolds (Fig. 7A, C, E and G).

The results obtained for the quantification of inflammation around the two types of SPCL scaffolds were identical (data not shown), and therefore only the results for the SPCL-WS scaffolds are described. The SPCL-WS scaffolds occupied an area which did not vary significantly from 8 to 12 weeks of implantation (Fig. 9). At 8 weeks of SC implantation the area of the inflammatory infiltrate was significantly lower than the area of the scaffolds (Fig. 9). However, the inflammation area notably increased from 8 to 12 weeks (Fig. 9) of implantation. Additionally, the inflammation area at 12 weeks of implantation was significantly higher compared with the area occupied by the implanted SPCL-WS scaffolds (Fig. 9).

Concerning the molecular expression of specific genes of inflammation, the SC implantation of both SPCL-WS and SPCL-FB scaffolds induced the expression of IL- $1 \alpha$, but not IL-18, both at 1 and 2 weeks of implantation (Table 2). Regarding the anti-inflammatory cytokines IL-10, IL-13, IFN- $\gamma$ and MHC class II, none of the tested conditions induced their expression. An exception was observed for the implantation of SPCL-WS for 2 weeks, which showed MHC II expression in half of the samples (Fig. 8 and Table 2).

In the long-term host reaction, the molecular biology analysis showed that the inflammatory marker IL- $1 \alpha$ was expressed at both 8 and 12 weeks of subcutaneous implantation of SPCL-WS and SPCL-FB scaffolds. Conversely IL-18 was not expressed under any conditions, except for the SPCL-FB implanted for 8 weeks (Table 2 ). It was expected that a long-term implantation reaction would result in a pro-wound healing pattern of cytokines, although this was not observed. Thus, after 8 weeks of SC implantation, both types of SPCL scaffolds induced the expression of IL-10, IL-13, IFN- $\gamma$ and MHC II, although half of the SPCL-FB samples did not induce the expression of IL-13 and IFN- $\gamma$ (Fig. 8 and Table 2). When SPCL-WS was implanted for 12 weeks, all the anti-inflammatory cytokines tested were expressed, albeit IFN- $\gamma$ was not expressed in half of the samples (Table 2). For the same time of implantation, the SPCL-FB scaffolds induced expression of IL-13 in all samples, but IFN- $\gamma$ and MHC II in only half of the samples (Table 2). On the contrary, IL-10 was not expressed after 12 weeks of SPCL-FB implantation (Fig. 8 and Table 2).

\section{Discussion}

In vivo studies with scaffolds intended for TE application are generally carried out with 3-D structures seeded, or not, with cells and with the addition of growth factors [11], in order to assess the role of the construct in the regeneration of a specific tissue; such studies are not aimed at addressing the host reaction. This research aimed to stress the influence and the relevance of the support material over the host reaction to an implanted TE construct, by addressing the responses elicited by the implantation of two different types of SPCL scaffolds.

Microscopically, SC implantation, considering the first two time periods, acute and onset of chronic inflammation, respectively, showed a slightly lower inflammatory reaction in comparison to IM implantation of both types of SPCL scaffolds. However, when the SPCL scaffolds were SC implanted for longer periods, the implant was perfectly integrated into the host tissue and the inflammatory process was resolving. In fact, the histological analysis at 8 and 12 weeks after SC implantation showed the absence of fibrotic capsule, lower amounts of FBGCs and a total inflammation area that was not significant in comparison to the area occupied by the scaffolds.

The presented results indicate that IM implantation of biomaterials may be considered a more reactive implantation model to evaluate biomaterial-host interaction in terms of inflammatory/ immune response, since the SC implantation showed a slightly lower inflammatory/immune reaction to the SPCL scaffolds.

The present work showed that SPCL scaffolds induced the expression of IL-18 when IM implanted both for 7 and 14 days. This means that macrophages are activated at the implantation site [36] The present results revealed a associated expression of IL-18 and IFN- $\gamma$, which is in accordance with a previous report demonstrating that IL-18 acts in T lymphocytes to induce the production of IFN- $\gamma$ [36], specifically at the second week of implantation. Conversely, the SC implantation of the SPCL scaffolds did not induce IL-18 expression and subsequent up-regulation of IFN- $\gamma$ secretion by $\mathrm{T}$ lymphocytes. This may indicate that the inflammatory process is being resolved with PMN apoptosis following activation [37]. However, IL- $1 \alpha$ is expressed at all times of implantation and in both implantation models. It is well known that, in the early stage of inflammation, IL- $1 \alpha$ is expressed by macrophages and endothelial cells, which thus stimulate activation of $\mathrm{B}$ and $\mathrm{T}$ lymphocytes, and at latter inflammation phase is secreted by dendritic cells and $B$ lymphocytes [36]. These phenomena explain the detection of IL$1 \alpha$ at an early stage expressed by macrophages and at a later stage secreted by B lymphocytes and dendritic cells. Beezhold and Lause [38] demonstrated that macrophage interaction with fibronectin can lead to an increased release of IL-1 cytokines and increased IL-1 mRNA expression. This may also be a reason for the constant presence of IL- $1 \alpha$ throughout all the implantation periods, indicated that macrophages were in contact with the fibronectin deposited on the SPCL scaffold surface $[28,39]$.

In the present work, IL-10 was expressed at 7 and 14 days of IM implantation, as well as at 8 weeks of SC implantation. Since IL-10 is secreted by $\mathrm{T}_{\mathrm{H}} 2$ lymphocytes and acts on antigen-presenting cells (APCs) by down-regulating MHC class II expression [36], our results showing the simultaneous expression of IL-10 and MHC class II were not expected. A possible reason for the observed expression patterns of the MHC class II encoding genes is the close correlation with IFN- $\gamma$ expression, since it was previously reported that the inhibition of IFN- $\gamma$ coincides with $\mathrm{MCH}$ class II inhibition [40].

With longer implantation periods, SPCL scaffolds induced the expression of IL-13, an important regulator of inflammation and a pro-wound cytokine [36]. This occurred as expected [41] and indicates that the surface chemistry of the SPCL scaffolds does not inhibit wound healing.

After 8 weeks of SC implantation of the SPCL scaffolds, the differences observed for the two different types of scaffolds were negligible, indicating that resolution of inflammation took place with 

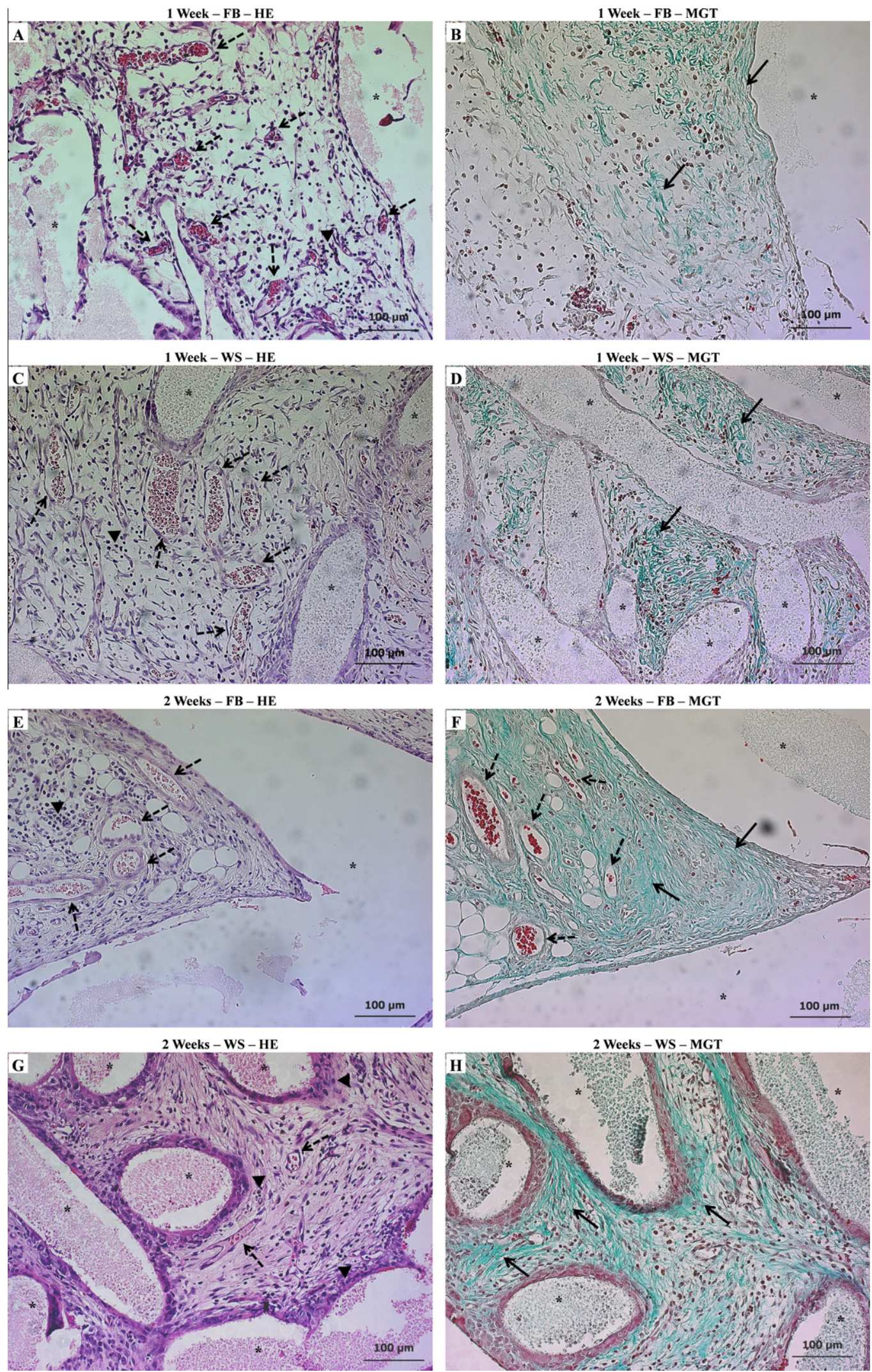

Fig. 4. Micrographs of the sections of the explanted FB and WS produced starch-based scaffolds, after 1 (A-D) and 2 (E-H) weeks of SC implantation in rats. Tissue was stained with HE (A, C, E and G) and MGT (B, D, F and G). $*$ represents the SPCL scaffold fibres. Inflammatory cells are marked with arrowheads, collagen fibres with arrows and blood vessels with dashed arrows.

the integration of the scaffolds in the host tissue. At week 12 after implantation, the induced cytokine expression profile was typical of chronic inflammatory process and normal progress of inflammation provoked by the implantation of biodegradable biomaterials, 

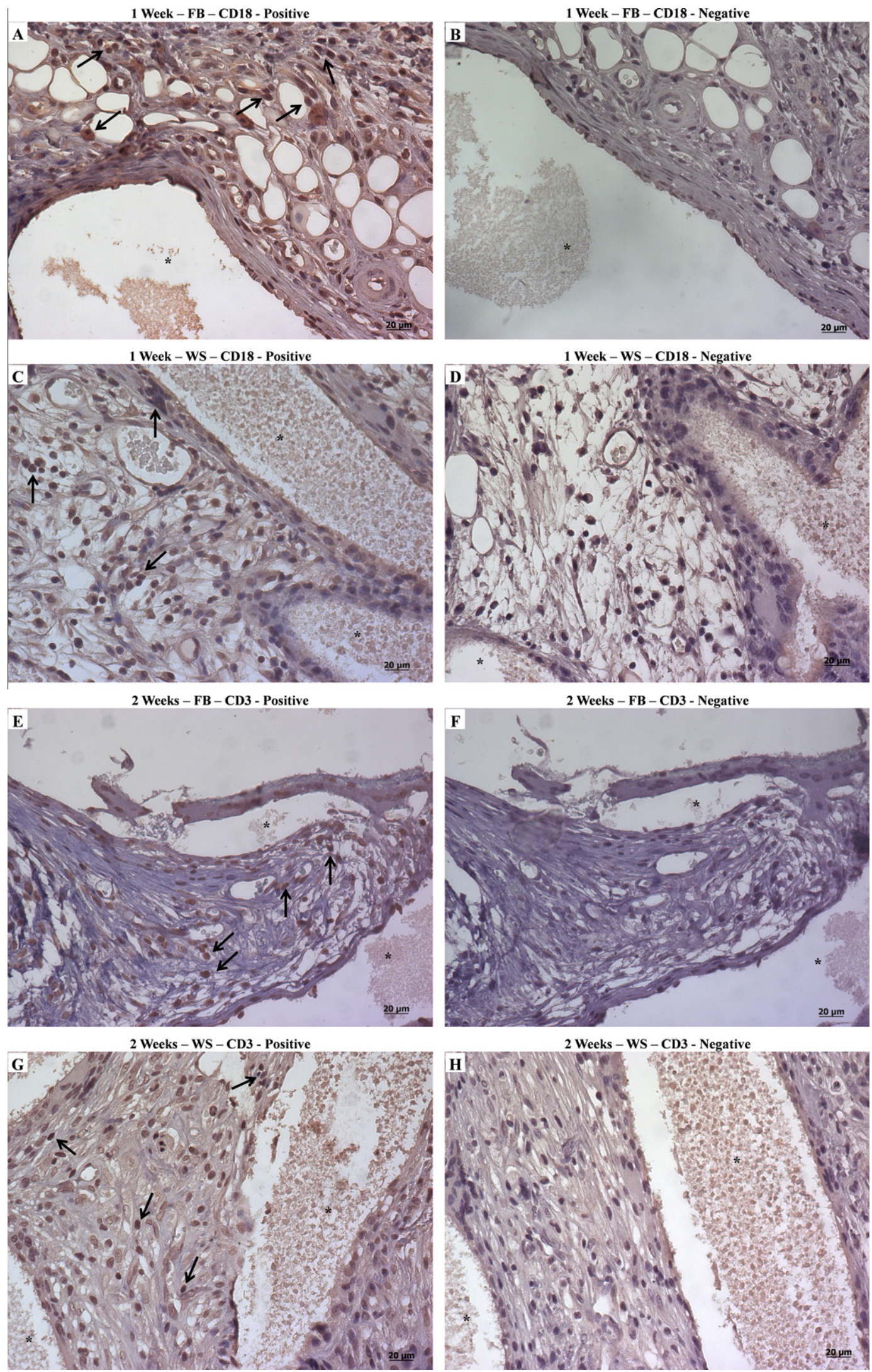

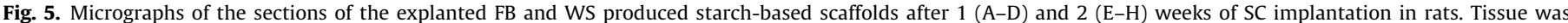
immunohistochemically labelled for CD18 and CD3. * represents the SPCL scaffold fibres, arrows mark CD18-positive cells and dashed arrows mark CD3-positive cells.

although no fibrotic capsule was histologically observed surrounding the fibres of the scaffolds.
The present results show that SPCL scaffolds produced by two different methodologies, WS [26] and FB [22] do not induce a 

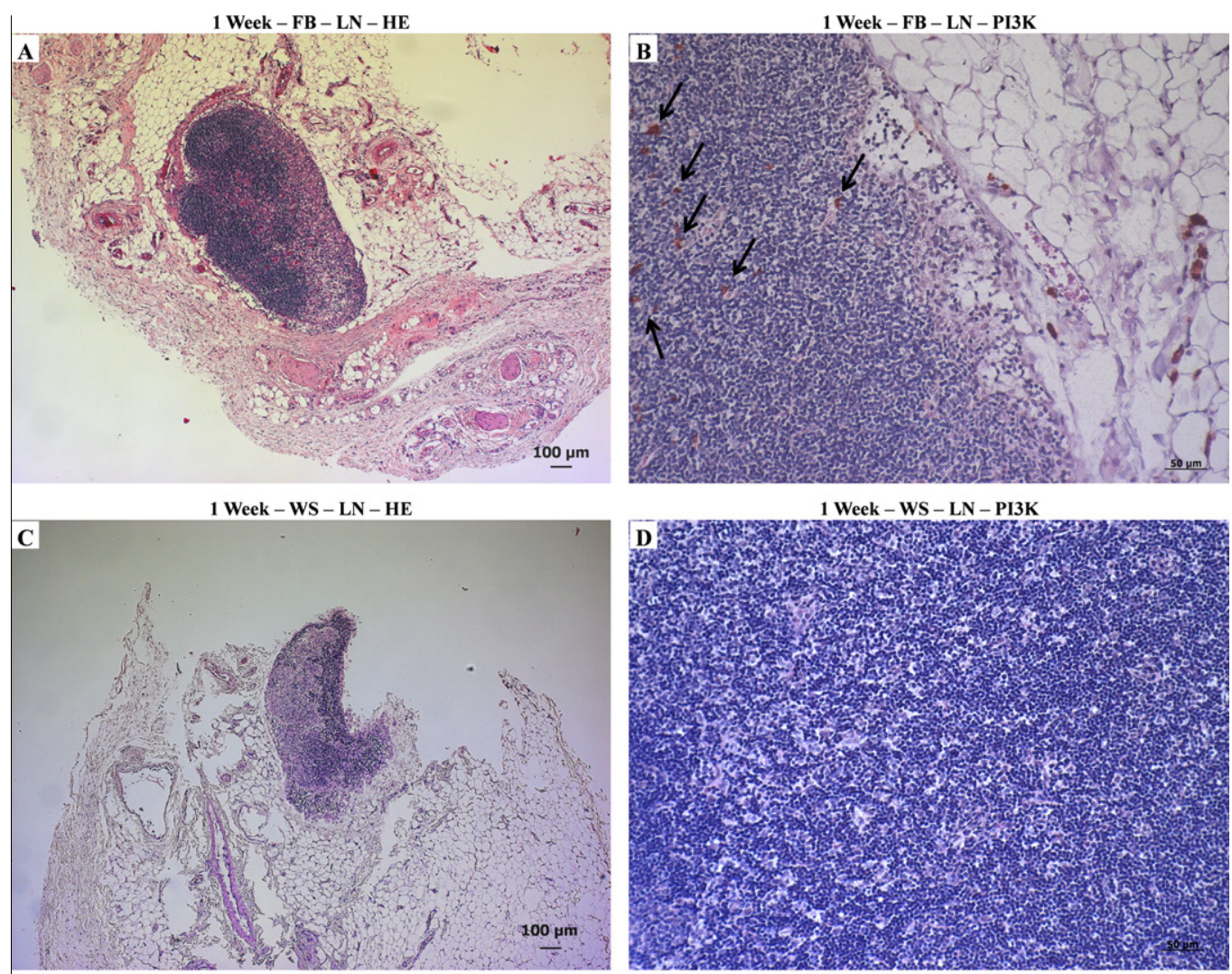

2 Weeks - FB - LN - HE
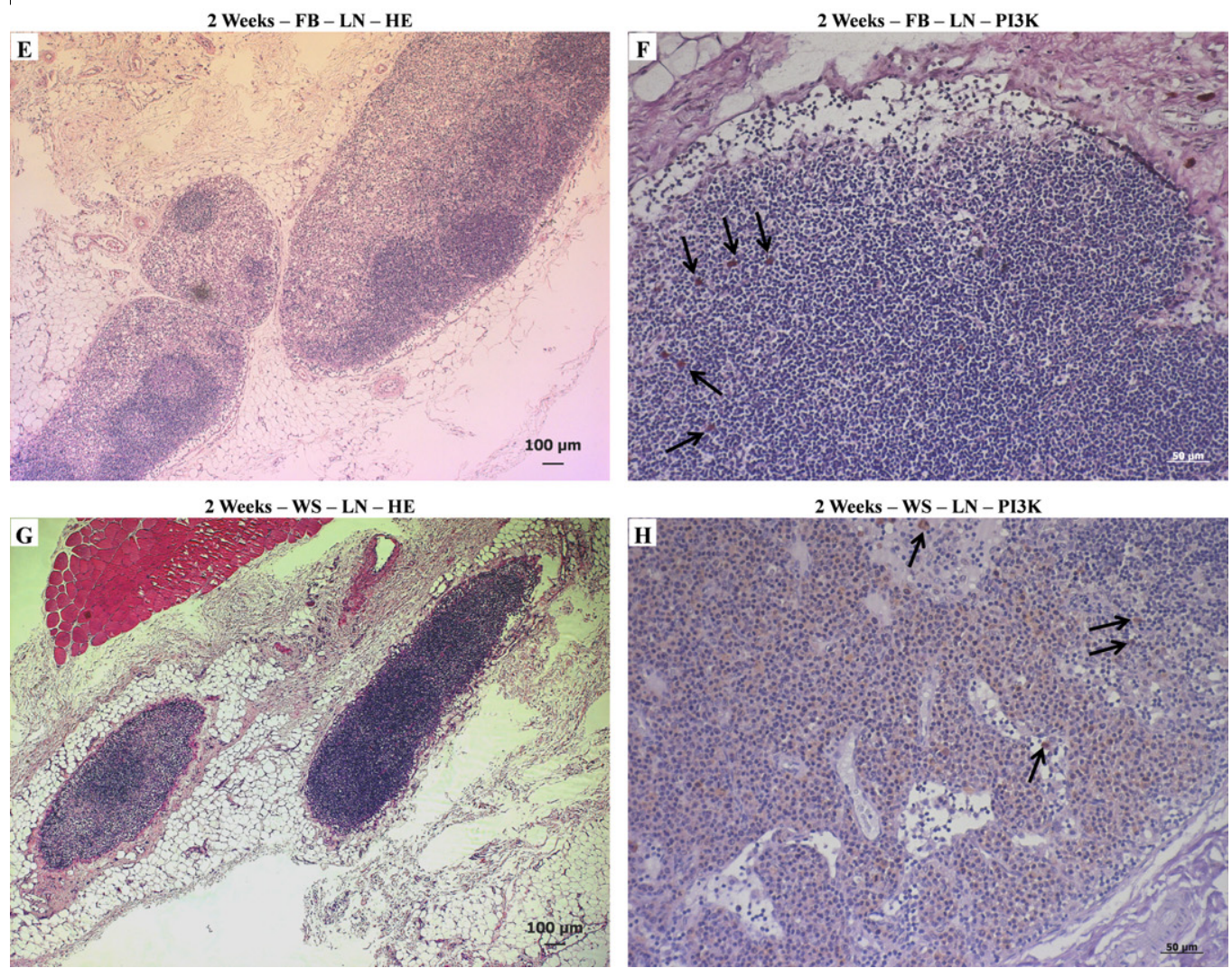

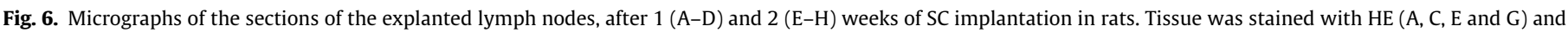
immunohistochemically labelled for Pi3K (B, D, F and G). Arrows indicate positive labelled cells for the PI3K transduction factor.

severe soft tissue inflammatory reaction. This could be observed by the low host reaction detected after SC and IM implantations of both types of SPCL scaffolds in rats for 1 and 2 weeks. Additionally, the a long-term implantation in the SC tissue of rats for 8 and 

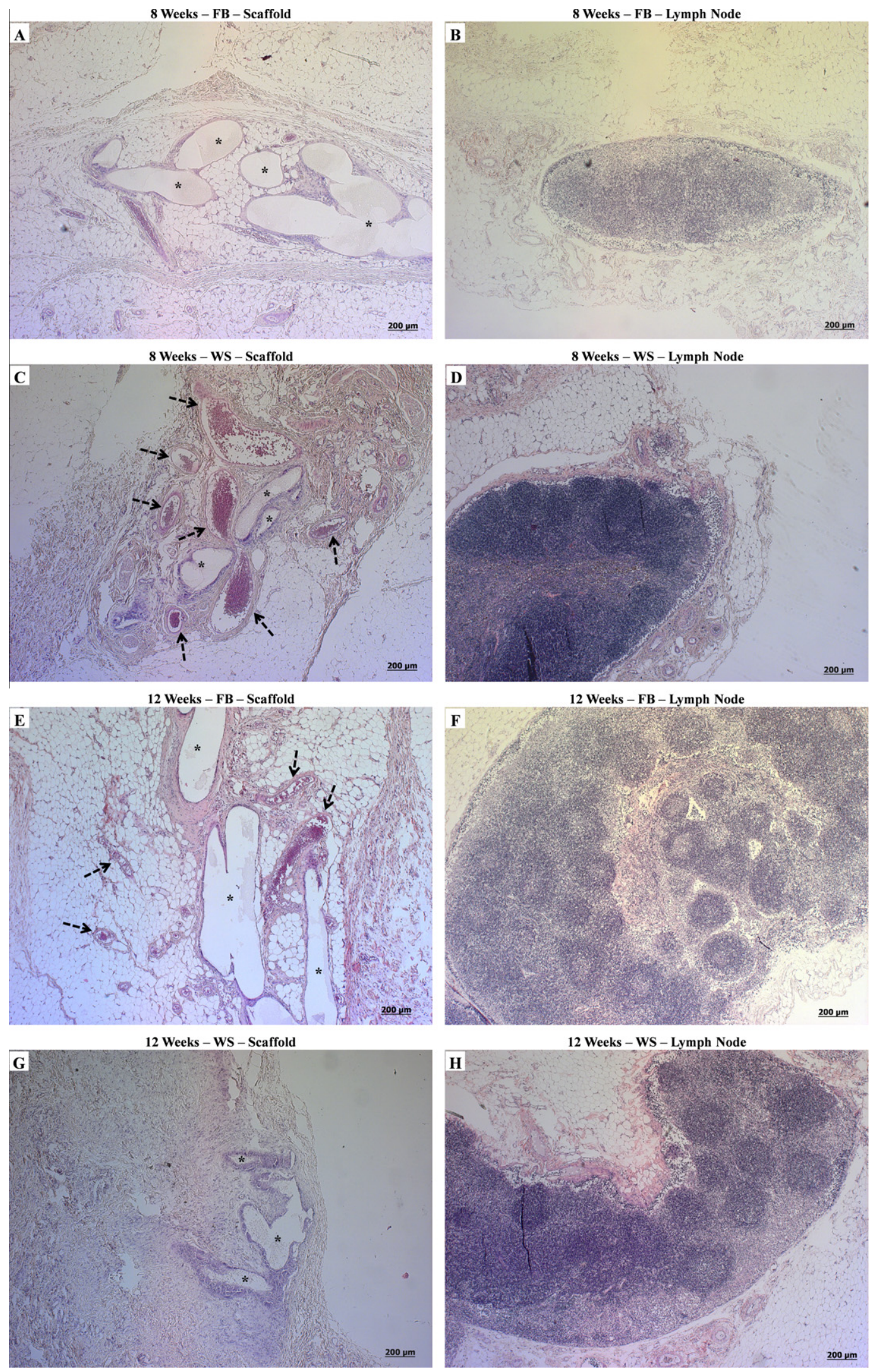

Fig. 7. Micrographs of the sections of the explanted FB and WS produced starch-based scaffolds (A, E, C and G) and nearby lymph nodes (B, F, D and H), after 8 (A-D) and 12 (E-H) weeks of SC implantation in rats. * represents the SPCL scaffold fibres. 


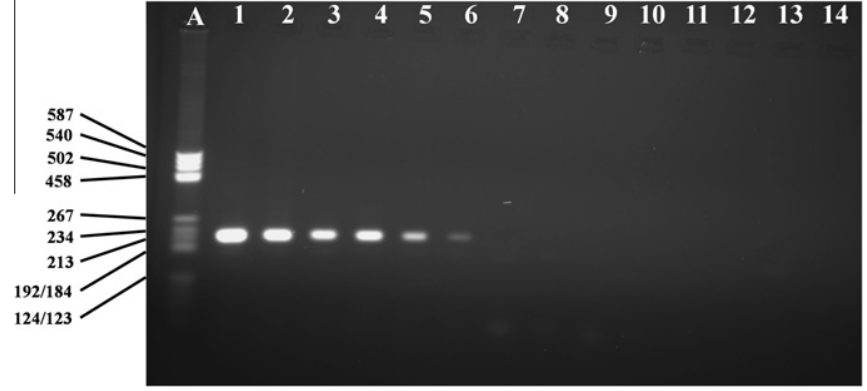

Fig. 8. Representative image of the electrophoresis gels reporting the results of the expression of the genes by RT-PCR. The gel shows the results obtained for the IL-10 gene expression after IM (1-6) and SC (7-13) SPCL scaffold implantation. Column 14 is the negative control and $A$ is the cDNA marker.

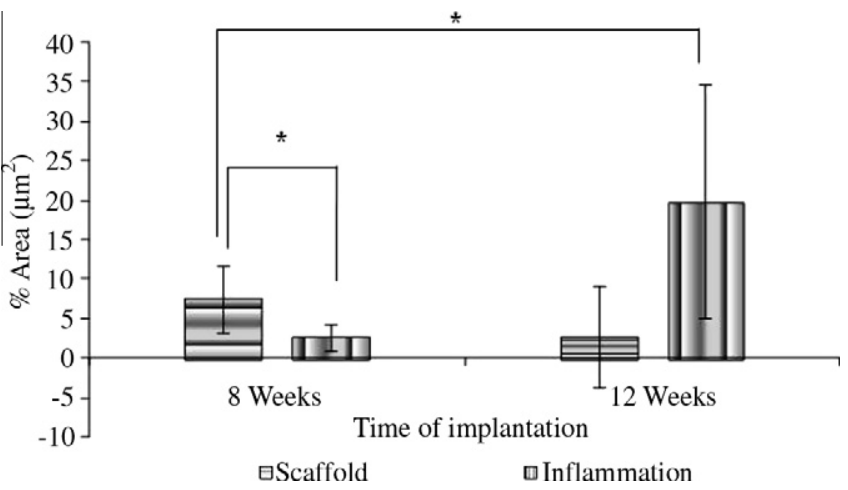

Fig. 9. Areas (mean \pm SD) occupied by the SPCL scaffolds and by the inflammatory infiltrate relatively to the total area, quantified on the micrographs. Significantly different at $P<0.05$.

12 weeks showed a good integration of the SPCL scaffolds into the host tissue and a pro-wound healing cytokine profile expression.

\section{Conclusions}

The present work demonstrates that SPCL-WS and SPCL-FB scaffolds induce a moderate inflammatory reaction after SC and IM implantations. Nevertheless, SPCL-WS seemed to be less reactive, particularly when the cytokine profile was evaluated, showing an early resolution of the inflammatory process compared with the SPCL-FB scaffolds.

Additionally, it was shown that IM implantation of the same type of materials induces a slightly more intense inflammatory response in comparison to the SC model, which may indicate that IM implantation is a more sensitive model to address the inflammation and immune host response to the implantation of a biomaterial.

\section{Acknowledgments}

T.C.S. acknowledges to the Marie Curie European Program for a short-term scholarship in the Alea Jacta EST project (MEST-CT2004-008104). This work was developed under the scope of the European Network of Excellence EXPERTISSUES (NMP3-CT-20045000283).

\section{Appendix A. Figures with essential colour discrimination}

Certain figures in this article, particularly Figures 1-7 are difficult to interpret in black and white. The full colour images can be found in the on-line version, at doi:10.1016/j.actbio.2010.06.020.

\section{References}

[1] Rada T, Reis RL, Gomes ME. Adipose tissue-derived stem cells and their application in bone and cartilage tissue engineering. Tissue Eng B Rev 2009;15:113-25.

[2] Rombouts WJ, Ploemacher RE. Primary murine MSC show highly efficient homing to the bone marrow but lose homing ability following culture. Leukemia 2003;17:160-70.

[3] Krebsbach PH, Mankani MH, Satomura K, Kuznetsov SA, Robey PG. Repair of craniotomy defects using bone marrow stromal cells. Transplantation 1998;66:1272-8.

[4] Bruder SP, Kurth AA, Shea M, Hayes WC, Jaiswal N, Kadiyala S. Bone regeneration by implantation of purified, culture-expanded human mesenchymal stem cells. J Orthop Res 1998;16:155-62.

[5] Bruder SP, Kraus KH, Goldberg VM, Kadiyala S. The effect of implants loaded with autologous mesenchymal stem cells on the healing of canine segmental bone defects. J Bone Joint Surg Am 1998;80:985-96.

[6] Bruder SP, Jaiswal N, Ricalton NS, Mosca JD, Kraus KH, Kadiyala S. Mesenchymal stem cells in osteobiology and applied bone regeneration. Clin Orthop Relat Res 1998:S247-56.

[7] Uebersax L, Merkle HP, Meinel L. Insulin-like growth factor I releasing silk fibroin scaffolds induce chondrogenic differentiation of human mesenchymal stem cells. J Control Release 2008;127:12-21.

[8] Abarrategi A, Civantos A, Ramos V, Sanz Casado JV, Lopez-Lacomba JL. Chitosan film as rhBMP2 carrier: delivery properties for bone tissue application. Biomacromolecules 2008;9:711-8.

9] Wei G, Jin Q Giannobile WV, Ma PX. The enhancement of osteogenesis by nano-fibrous scaffolds incorporating rhBMP-7 nanospheres. Biomaterials 2007;28:2087-96.

[10] Jeon O, Song SJ, Kang SW, Putnam AJ, Kim BS. Enhancement of ectopic bone formation by bone morphogenetic protein-2 released from a heparin-conjugated poly(L-lactic-co-glycolic acid) scaffold. Biomaterials 2007;28:2763-71.

[11] Mikos AG, McIntire LV, Anderson JM, Babensee JE. Host response to tissue engineered devices. Adv Drug Deliv Rev 1998;33:111-39.

[12] Aggarwal S, Pittenger MF. Human mesenchymal stem cells modulate allogeneic immune cell responses. Blood 2005;105:1815-22.

[13] Rasmusson I, Ringden O, Sundberg B, Le Blanc K. Mesenchymal stem cells inhibit lymphocyte proliferation by mitogens and alloantigens by different mechanisms. Exp Cell Res 2005;305:33-41.

[14] Glennie S, Soeiro I, Dyson PJ, Lam EW, Dazzi F. Bone marrow mesenchymal stem cells induce division arrest anergy of activated $\mathrm{T}$ cells. Blood 2005; $105: 2821-7$

[15] Niemeyer P, Kornacker M, Mehlhorn A, Seckinger A, Vohrer J, Schmal H, et al. Comparison of immunological properties of bone marrow stromal cells and adipose tissue-derived stem cells before and after osteogenic differentiation in vitro. Tissue Eng 2007;13:111-21.

[16] Puissant B, Barreau C, Bourin P, Clavel C, Corre J, Bousquet C, et al. Immunomodulatory effect of human adipose tissue-derived adult stem cells: comparison with bone marrow mesenchymal stem cells. $\mathrm{Br} \mathrm{J}$ Haematol 2005;129:118-29.

[17] Wolbank S, Peterbauer A, Fahrner M, Hennerbichler S, van Griensven M, Stadler G, et al. Dose-dependent immunomodulatory effect of human stem cells from amniotic membrane: a comparison with human mesenchymal stem cells from adipose tissue. Tissue Eng 2007;13:1173-83.

[18] Crouzier T, Ren K, Nicolas C, Roy C, Picart C. Layer-by-layer films as biomimetic reservoir for rhBMP-2 delivery: controlled differentiation of myoblasts to osteoblasts. Small 2009;5:598-608.

[19] Gomes ME, Ribeiro AS, Malafaya PB, Reis RL, Cunha AM. A new approach based on injection moulding to produce biodegradable starch-based polymeric scaffolds: morphology, mechanical and degradation behaviour. Biomaterials 2001;22:883-9.

[20] Gomes ME, Bossano CM, Johnston CM, Reis RL, Mikos AG. In vitro localization of bone growth factors in constructs of biodegradable scaffolds seeded with marrow stromal cells and cultured in a flow perfusion bioreactor. Tissue Eng 2006;12:177-88.

[21] Salgado AJ, Coutinho OP, Reis RL, Davies JE. In vivo response to starch-based scaffolds designed for bone tissue engineering applications. J Biomed Mater Res A 2007;80A:983-9.

[22] Gomes ME, Azevedo HS, Moreira AR, Ella V, Kellomaki M, Reis RL Starchpoly(epsilon-caprolactone) and starch-poly(lactic acid) fibre-mesh scaffolds for bone tissue engineering applications: structure, mechanical properties and degradation behaviour. J Tissue Eng Regen Med 2008;2:243-52.

[23] Santos MI, Tuzlakoglu K, Fuchs S, Gomes ME, Peters K, Unger RE, et al. Endothelial cell colonization and angiogenic potential of combined nano- and micro-fibrous scaffolds for bone tissue engineering. Biomaterials 2008;29:4306-13.

[24] Azevedo HS, Reis RL. Encapsulation of alpha-amylase into starch-based biomaterials: an enzymatic approach to tailor their degradation rate. Acta Biomater 2009;5:3021-30.

[25] Martins AM, Pham OP, Malafaya PB, Sousa RA, Gomes ME, Raphael RM, et al. The role of lipase and alpha-amylase in the degradation of starch/poly(varepsiloncaprolactone) fiber meshes and the osteogenic differentiation of cultured marrow stromal cells. Tissue Eng A 2009;15:295-305.

[26] Tuzlakoglu K, Pashkuleva I, Rodrigues MT, Gomes ME, van Lenthe GH, Müller $\mathrm{R}$, et al. A new route to produce starch-based fiber mesh scaffolds by wet 
4326

T.C. Santos et al./Acta Biomaterialia 6 (2010) 4314-4326

spinning and subsequent surface modification as a way to improve cell attachment and proliferation. J Boomed Mater Res A 2010;92:369-77.

[27] Marques AP, Cruz HR, Coutinho OP, Rein RL. Effect of starch-based biomaterials on the in vito proliferation and viability of osteoblast-like cells. J Mater Sci Mater Med 2005;16:833-42.

[28] Alves CM, Yang Y, Carne DL, Ing JL, Sylvia VL, Dean DD, et al. Modulating bone cells response onto starch-based biomaterials by surface plasma treatment and protein adsorption. Biomaterials 2007;28:307-15.

[29] Balmayor ER, Tuzlakoglu K, Marques AP, Azevedo HS, Reis RL. A novel enzymatically-mediated drug delivery carrier for bone tissue engineering applications: combining biodegradable starch-based microparticles and differentiation agents. J Mater Sci Mater Med 2008;19:1617-23.

[30] Martins AM, Santos MI, Azevedo HS, Malafaya PB, Reis RL. Natural origin scaffolds with in situ pore forming capability for bone tissue engineering applications. Acts Biomater 2008;4:1637-45.

[31] Azevedo HS, Gama FM, Reis RL. In vito assessment of the enzymatic degradation of several starch based biomaterials. Biomacromolecules 2003;4:1703-12.

[32] Santos MI, Unger RE, Sousa RA, Reis RL, Kirkpatrick CJ. Crosstalk between osteoblasts and endothelial cells co-cultured on a polycaprolactone-starch scaffold and the in vitro development of vascularization. Biomaterials 2009;30:4407-15.
[33] Santos MI, Fuchs S, Gores ME, Unger RE, Reis RL, Kirkpatrick CJ. Response of micro- and macrovascular endothelial cells to starch-based fiber meshes for bone tissue engineering. Biomaterials 2007;28:240-8.

[34] Reis RL, Menders SC, Cunha AM, Bevis ML. Processing and in vito degradation of starch/EVOH thermoplastic blends. Polym Int 1997;43:347.

[35] Kirkwood B, Sterne J. Essential medical statistics. Wiley; 2003.

[36] Goldsby RA, Kind TJ, Osborne BA. Kuby immunology. New York: W.H. Freeman; 2000.

[37] Kobayashi SD, Voyich JM, Braughton KR, DeLeo FR. Down-regulation of proinflammatory capacity during apoptosis in human polymorphonuclear leukocytes. J Immunol 2003;170:3357-68.

[38] Beezhold DH, Laue DB. Stimulation of rat macrophage interleukin 1 secretion by plasma fibronectin. Immunol Invest 1987;16:437-49.

[39] Alves CM, Reis RL, Hunt JA. Preliminary study on human protein adsorption and leukocyte adhesion to starch-based biomaterials. J Mater Sci Mater Med 2003; 14:157-65.

[40] Khoum IM, van Wachem PB, de Leif LF, van Luyn MJ. Inhibition of the tissue reaction to a biodegradable biomaterial by monoclonal antibodies to IFNgamma. J Boomed Mater Res 1998;41:202-10.

[41] Brodbeck WG, Voskerician G, Ziats NP, Nakayama Y, Matsuda T, Anderson JM. In vino leukocyte cytokine ERNA responses to biomaterials are dependent on surface chemistry. J Boomed Mater Res A 2003;64:320-9. 